\title{
Terapia de Casal e Estratégias de Resolução de Conflito: Uma Revisão Sistemática
}

\author{
Crístofer Batista da Costa \\ Universidade do Vale do Rio dos Sinos, RS, Brasil. \\ Adriana Wagner \\ Universidade Federal do Rio Grande do Sul, RS, Brasil.
}

\author{
Marina Zanella Delatorre \\ Universidade Federal do Rio Grande do Sul, RS, Brasil. \\ Clarisse Pereira Mosmann \\ Universidade do Vale do Rio dos Sinos, RS, Brasil.
}

Resumo: O objetivo deste estudo foi realizar uma revisão sistemática de artigos científicos sobre psicoterapia de casal e estratégias de resolução de conflito e identificar variáveis envolvidas no processo terapêutico. Foram consultadas oito bases de dados: Academic Search Complete, Biblioteca Cochrane, Ibecs, Lilacs, Medline, PsycINFO, SciELO e Web of Science, no período de 2006 a 2015. Foram encontrados 238 artigos, sendo incluídos 13 estudos após a avaliação de três juízes independentes. As abordagens comportamental e cognitivo-comportamental se destacaram em relação à sistêmica e à psicodinâmica. Observou-se consenso sobre o papel da comunicação, da empatia, do perdão, do apoio mútuo e da confiança para a resolução dos conflitos conjugais. Por outro lado, há falta de homogeneidade e de clareza na utilização de conceitos e técnicas. A análise da literatura forneceu um panorama internacional, composto por perspectivas de consenso já estabelecidos, inconsistências que devem ser superadas e carência de estudos, especialmente no Brasil, contexto potencial de investigação da temática. Palavras-chave: Casamento, Conflito Conjugal, Terapia de Casal, Resolução de Problemas.

\section{Couple Therapy and Conflict Resolution Strategies: A Systematic Review}

\begin{abstract}
The aim of this study was to conduct a systematic review of scientific articles about couple psychotherapy and conflict resolution strategies and to identify variables involved in the therapeutic process. Eight databases were consulted: Academic Search Complete, Cochrane Library, IBECS, Lilacs, Medline, PsycINFO, SciELO and Web of Science, from 2006 to 2015. We found 238 articles and, after an evaluation by three independent judges, 13 studies were included in this review. The behavioral and cognitive-behavioral approaches have excelled in relation to the systemic and to the psychodynamic ones. On one hand, there was consensus on the role of communication, empathy, forgiveness, mutual support and confidence for resolving marital conflicts. On the other hand, there is a lack of homogeneity and clarity in the use of the concepts and techniques. The literature review provided an international overview, consisting of consensual perspectives already established, inconsistencies that must be overcome and a lack of studies, especially in Brazil, culminating in a potential context of thematic research.
\end{abstract}

Keywords: Marriage, Marital Conflict, Couple Therapy, Problem Solving. 


\title{
Terapia de Pareja y Estrategias de Resolución de Conflicto: Una Revisión Sistemática
}

\begin{abstract}
Resumen: El objetivo de este estudio fue realizar una revisión sistemática de artículos científicos acerca de la psicoterapia de pareja y estrategias de resolución de conflictos, así como identificar variables involucradas en el proceso terapéutico. Se consultaron ocho bases de datos: Academic Search Complete, Biblioteca Cochrane, Ibecs, Lilacs, Medline, PsycINFO, SciELO y Web of Science, en el período de 2006 a 2015. Fueron encontrados 238 artículos; fueron incluidos 13 estudios después de la evaluación de tres jueces independientes. Los abordajes comportamental y cognitivo-conductual se destacaron en relación al sistémico y psicodinámico. Se observó un consenso acerca del rol de la comunicación, de la empatía, del perdón, del apoyo mutuo y de la confianza para la resolución de los conflictos conyugales. Por otro lado, hay falta de homogeneidad y claridad en la utilización de conceptos y técnicas. El análisis de la literatura proporcionó un panorama internacional, compuesto por perspectivas de consenso ya establecidas, incongruencias que deben ser superadas y carencia de estudios, en especial en Brasil, contexto potencial de investigación de esta temática.
\end{abstract}

Palabras clave: Matrimonio, Conflicto Conyugal, Terapia de Pareja, Resolución de Problemas.

\section{Introdução}

Conflitos conjugais vêm sendo investigados na literatura internacional há cerca de cinco décadas (Fincham, 2003; Mitchell, Bullard, \& Mudd, 1962), abrangendo seus motivos, intensidade, frequência e forma de encaminhamento (Costa, Cenci, \& Mosmann, 2016; Mosmann, \& Falcke, 2011; Scheeren, Delatorre, Neumann, \& Wagner, 2015). As estratégias que os cônjuges utilizam para resolver seus conflitos predizem se o desfecho da situação será mais ou menos efetivo (Schoebi, Karney, \& Bradbury, 2012; Veldorale-Brogan, Lambert, Fincham, \& DeWall, 2013). Essas estratégias são comportamentos adotados com a finalidade de encontrar uma solução para o problema, pressupõem a necessidade de negociação entre os parceiros e são consideradas construtivas ou destrutivas (Carlson, \& Dinkmeyer, 1987; Leggett, Roberts-Pittman, Byczek, \& Morse, 2012; Schoebi et al., 2012).

As estratégias construtivas envolvem comunicação respeitosa, percepção do conflito como oportunidade de melhorar o relacionamento, clareza sobre a corresponsabilidade dos cônjuges, autocontrole, flexibilidade, tolerância e a busca conjunta por uma solução satisfatória para o casal. As destrutivas, por sua vez, caracterizam-se pela identificação de culpados, foco demasiado no problema, racionalização, comportamentos de esquiva e de retraimento, hostilidade, reclamações, rigidez e negatividade (Anderson \& Johnson, 2010; Sierau,
\& Herzberg, 2012; Sullivan, Pasch, Johnson, \& Bradbury, 2010; Wheeler, Updegraff, \& Thayer, 2010; Whiting, 2008).

A comunicação e a resolução de conflitos na conjugalidade foram investigadas por Markman, Rhoades, Stanley, Ragan e Whitton (2010), em um estudo com 210 casais americanos. Os pesquisadores avaliaram associações entre a comunicação e os conflitos conjugais antes do casamento e nas situações em que ocorreu divórcio nos primeiros cinco anos de união. Os resultados evidenciaram o que os autores denominaram o "efeito da negatividade", isto é, a comunicação negativa é um fator de risco mais forte, tanto para o conflito quanto para o divórcio, do que a comunicação positiva é um fator de proteção. Os autores argumentam que experiências negativas são mais dolorosas e, por isso, os cônjuges tendem a supervalorizá-las em detrimento das positivas. Modelos negativos de resolução de conflito na família de origem, por outro lado, podem se repetir nos relacionamentos conjugais dos descendentes. Isso aconteceria, principalmente, com casais que possuem baixa capacidade de identificar os padrões interacionais e comportamentais aprendidos da relação conjugal dos pais que se repetem no próprio casamento (Curran, Ogolsky, Hazen, \& Bosch, 2011). Nesse sentido, a psicoterapia poderia auxiliar o casal a identificar estilos destrutivos de resolver conflitos aprendidos na família de origem e alterar padrões de comunicação negativa. 
Embora o foco das pesquisas em psicoterapia seja a clínica individual e os estudos de caso único, nas metodologias quantitativa e qualitativa e dentro das diversas abordagens teóricas (Brum, Frizzo, Gomes, Silva, Souza, \& Piccinini, 2012; Enéas, 2008; Peuker, Habigzang, Koller, \& Araujo, 2009), a psicoterapia de casal vem sendo investigada, ainda que mais timidamente, há aproximadamente quatro décadas. Os primeiros estudos sobre terapia de casal encontrados na literatura científica datam da década de 1970 (Jacobson, 1978; 1979). Essas pesquisas investigavam a efetividade do treinamento para resolução de conflitos com casais que tinham conflitos graves e comparavam as estratégias adotadas por casais em terapia com aquelas de casais que aguardavam em lista de espera. As díades em tratamento demonstravam maior capacidade de resolver os conflitos se comparadas com as que ainda não haviam iniciado psicoterapia, tais mudanças se mantinham ao longo do tempo.

Nos anos 1980, foi produzido um número expressivo de estudos sobre terapia de casal na perspectiva comportamental (Baucom, 1982; Jacobson, 1978; 1979; 1984; Jacobson, Follette, Follette, Holtzworth-Munroe, Katt, \& Schmaling, 1985; Jacobson, Schmaling, \& Holtzworth-Munroe, 1987; Sayers, Baucom, Sher, \&Weiss, 1991). As pesquisas nesta área focavam, principalmente, a resolução de conflitos, a comunicação conjugal, a satisfação com o relacionamento, o engajamento na terapia e os comportamentos positivos nas situações envolvendo conflitos graves. O foco era avaliar a eficácia da psicoterapia utilizando medidas pré e pós-tratamento e de follow-up por meio de critérios metodológicos rigorosos.

Nos anos 1990 e início dos anos 2000, foram produzidos estudos à luz da teoria cognitivo-comportamental (Behrens, Sanders, \& Halford, 1990; Braukhaus, Hahlweg, Kroeger, Groth, \& Fehm-Wolfsdorf, 2003; Kaiser, Hahlweg, Fehm-Wolfsdorf, \& Groth, 1998) e da teoria sistêmica (Davidson, \& Horvath, 1997; Goldman, \& Greenberg, 1992; Johnson, \& Greenberg, 1985; Rohrbaugh, \& Shoham, 2001). Os estudos da primeira abordagem teórica investigavam os efeitos dos programas de prevenção e das técnicas de psicoeducação, individuais e em grupo, além de comparar os resultados de diferentes abordagens, como a Terapia Conjugal Comportamental (BMT) e a Terapia Conjugal Cognitivo-comportamental (CBMT). Na perspectiva sistêmica, também eram comparadas diferentes abordagens, como a Terapia Sistêmica Integrativa (IST) e a Terapia
Focada na Emoção (EFT), utilizando medidas pré e pós-psicoterapia e de follow-up.

Nessa perspectiva, observa-se que nas últimas quatro décadas foram desenvolvidos estudos de eficácia, de efetividade e de processo. Os estudos de eficácia avaliam a validade interna da psicoterapia em ambiente controlado utilizando critérios de inclusão e exclusão rigorosos, amostra randomizada, protocolos, treinamento e acompanhamento para os terapeutas. Os estudos de efetividade avaliam a validade externa no contexto real onde o tratamento ocorre, caracterizam-se como quase experimentais por investigar a prática clínica com especificidades que não se pode controlar, como por exemplo, comorbidades, heterogeneidade da amostra, diferenças entre os profissionais (Peuker et al., 2009; Serralta, Nunes, \& Eizirik, 2011). Os estudos de processo em psicoterapia investigam o que ocorre durante o tratamento e as variáveis associadas à mudança no paciente. São analisados fatores inespecíficos nos tratamentos psicológicos, como a aliança terapêutica e a relação terapeuta-paciente, e fatores específicos, como os processos de mudança e os comportamentos do terapeuta que levaram à mudança. Tais alterações são apontadas como uma alternativa para se superar o veredito Dodô, termo utilizado na década de 1970 defendendo a equivalência entre as terapias no que se referia aos resultados satisfatórios dos tratamentos psicoterápicos, isto é, não se conseguia demonstrar diferenças significativas entre elas, apenas se evidenciava que funcionavam (Brum et al., 2012; Serralta et al., 2011).

De modo geral, as pesquisas em psicoterapia têm como objetivo identificar, testar, desenvolver e, sobretudo, estimular o uso e a disseminação de técnicas psicoterápicas válidas cientificamente visando a saúde mental dos indivíduos (Brum et al., 2012). Esses estudos avaliam se as psicoterapias produzem resultados efetivos, quais fatores e de que forma favorecem a remissão de sintomas. Além disso, possibilitam o desenvolvimento fundamentado da prática profissional e demonstram que pesquisa e clínica são, portanto, indissolúveis e complementares (Enéas, 2008; Peuker et al., 2009; Serralta et al., 2011).

Nesse sentido, foram considerados para a realização dessa revisão: a relevância das pesquisas baseadas em evidências, a escassez desses estudos com casais e o foco das pesquisas em psicoterapia conjugal nas estratégias de resolução de conflito, variável central no processo terapêutico e fundamental para a satis- 
fação e a qualidade do casamento em longo prazo (Leggett et al., 2012; Paleari, Regalia, \& Fincham, 2010; Schoebi et al., 2012; Veldorale-Brogan et al., 2013). Frente a isso, o objetivo neste estudo foi realizar uma revisão sistemática de artigos científicos sobre psicoterapia de casal e estratégias de resolução de conflito e identificar variáveis envolvidas no processo terapêutico. Conhecer como se configura o panorama dos estudos sobre a temática favorecerá identificar lacunas e novas perspectivas de investigação. Ademais, analisar e discutir modelos teóricos e diferentes técnicas de resolução de conflito para o atendimento das demandas conjugais na clínica poderá contrapor as variações entre abordagem teórica, técnica e demanda que influenciam o processo psicoterápico.

\section{Método}

Delineamento: trata-se de uma revisão sistemática da literatura de natureza descritiva em que foram feitas síntese e análise crítica do material levantado por meio de procedimentos sistemáticos, explícitos e replicáveis, cujo objetivo foi integrar, descrever, apontar divergências e convergências e orientar novas direções às pesquisas da área (Costa, Zoltowski, Koller, \& Teixeira, 2015; Sampaio, \& Mancini, 2007). Os procedimentos realizados neste estudo foram: a) considerações sobre o estado da arte da temática investigada; b) definição dos critérios de inclusão e exclusão; c) análise das características e dos resultados dos estudos elegidos; d) apresentação dos resultados, discussão e conclusões (Sampaio, \& Mancini, 2007).

Procedimentos de busca: a pesquisa foi realizada em oito bases de dados: Lilacs, Ibecs, Medline, Biblioteca Cochrane e SciELO (via Portal da Biblioteca Virtual em Saúde - BVS), Academic Search Complete, PsycINFO e Web of Science. Os termos de busca foram definidos de acordo com os descritores em Ciências da Saúde (Decs/BVS) e foi empregado o operador boleano AND entre os termos. Os descritores em português e inglês foram: 1) terapia de casal AND resolução de problemas - couples therapy AND problem solving e 2) terapia de casal AND resolução de conflitos - couples therapy AND conflict resolution.

Os procedimentos de busca, seleção e análise dos artigos foram realizados por dois juízes independentes em cinco etapas e de acordo com os critérios utilizados na escala para avaliação da qualidade metodológica das revisões sistemáticas (Costa et al., 2015).
Etapa (1): busca, nas bases de dados, de artigos científicos das ciências humanas e da saúde, nos idiomas português, espanhol e inglês, disponíveis em texto completo, publicados entre 2006 e 2015 - a busca foi realizada em agosto de 2015 e continha ao menos um dos seguintes unitermos no título "terapia de casal", "resolução de conflitos" ou "resolução de problemas"; Etapa (2): exclusão dos estudos repetidos entre as bases de dados; Etapa (3): análise dos resumos, que deveriam indicar pesquisa empírica e abranger pelo menos uma das temáticas descritas na etapa 1. Foram excluídas, nesta etapa, dissertações, teses, artigos teóricos, meta-análises e estudos cujo foco era individual, psicoeducativo ou envolviam a análise de outros fatores, como doença crônica e comorbidades psiquiátricas; Etapa (4): verificação da concordância entre juízes quanto à exclusão ou inclusão de estudos e encaminhamento dos casos discordantes para avaliação de um terceiro juiz e realização de consenso; Etapa (5): elegibilidade, descrição, análise e discussão dos estudos pelos juízes.

Análise dos dados: os estudos foram analisados a partir da sua abordagem teórica, delineamento, ano de publicação, local de realização, objetivos e principais resultados. Foi realizada uma leitura horizontal dos manuscritos, verificando-se a presença de variáveis comuns entre as diferentes perspectivas teóricas, técnicas ou propostas de intervenção. Posteriormente, uma avaliação vertical, analisando-se variáveis de investigação específicas em cada estudo.

\section{Resultados}

A busca nas bases de dados resultou em 238 artigos, conforme Figura: Academic Search Complete ( $n=39)$, Portal da Biblioteca Virtual em Saúde $(n=55)$, PsycINFO $(n=64)$ e Web of Science $(n=80)$. Destes 238 estudos, foram excluídos 170 , por não constar no título nenhum dos unitermos definidos, e 20 repetidos entre as bases de dados. Na etapa seguinte, cada juiz analisou separadamente os 48 estudos restantes. Houve concordância na análise de 43 artigos, sendo 35 para exclusão e oito considerados pertinentes à proposta deste estudo.

Os artigos excluídos foram: estudos teóricos, meta-análises e pesquisas que analisaram outras variáveis no casamento, tais como, depressão, câncer, alcoolismo ou uso de substâncias psicoativas, investigação das estratégias em uma perspectiva indivi- 
dual, intervenção psicoeducativa com casais e orientação para conflitos conjugais via internet. Houve discordância entre os juízes sobre cinco estudos que, embora investigassem resolução de conflito e psicoterapia de casal, envolviam propostas específicas de determinados grupos de pesquisadores sobre as variáveis perdão e apego, associadas às estratégias. Estes artigos foram avaliados por um terceiro juiz e, então, decidiu-se consensualmente pela inclusão de todos.

Foi analisada uma amostra total de 13 artigos, descritos na Tabela 1. Percebe-se que a maioria dos estudos foi publicado em 2011, sendo a maior parte nos Estados Unidos $(n=7)$, seguido do Canadá $(\mathrm{n}=4)$, Holanda $(\mathrm{n}=1)$ e Noruega $(\mathrm{n}=1)$. A abordagem teórica mais frequente foi a Comportamental e a Cognitivo-comportamental, seguida pela Sistêmica e pesquisas que utilizaram mais de uma teoria. Os delineamentos mais utilizados foram os ensaios clínicos randomizados e os estudos clínicos sem grupo controle. Foram encontrados também estudos de caso, estudos clínicos com grupo controle não randomizado e estudos de observação. Cabe ressaltar que os ensaios clínicos randomizados apresentaram amostras maiores e foram mais frequentes na abordagem compor- tamental. Já os estudos clínicos sem grupo controle foram mais frequentes na abordagem sistêmica.

Na Tabela 2 são apresentados os objetivos e os resultados dos estudos analisados. Três categorias de objetivos foram identificadas: a) Foco na teoria, em que diferentes abordagens foram comparadas; b) Foco em uma temática associada à resolução dos conflitos, nas quais foram identificadas distintas variáveis associadas às estratégias de resolução durante a terapia; e c) Foco na abordagem teórica e em uma temática associada à resolução dos conflitos.

Quanto aos resultados, observa-se que os estudos baseados nas teorias comportamentais reportaram aumento no ajustamento conjugal, na regulação emocional, na comunicação, nos comportamentos positivos e na resolução dos conflitos. Houve também diminuição dos sintomas depressivos e da expressão de raiva. Nessa abordagem, a Terapia Comportamental Tradicional de Casal (TBCT) demonstrou resultados superiores em relação à Terapia Comportamental Integrativa de Casal (IBCT) durante o tratamento, atribuídos, principalmente, ao acompanhamento do terapeuta. Porém, os resultados não se mantiveram após o término do tratamento. Outros estudos demonstra-

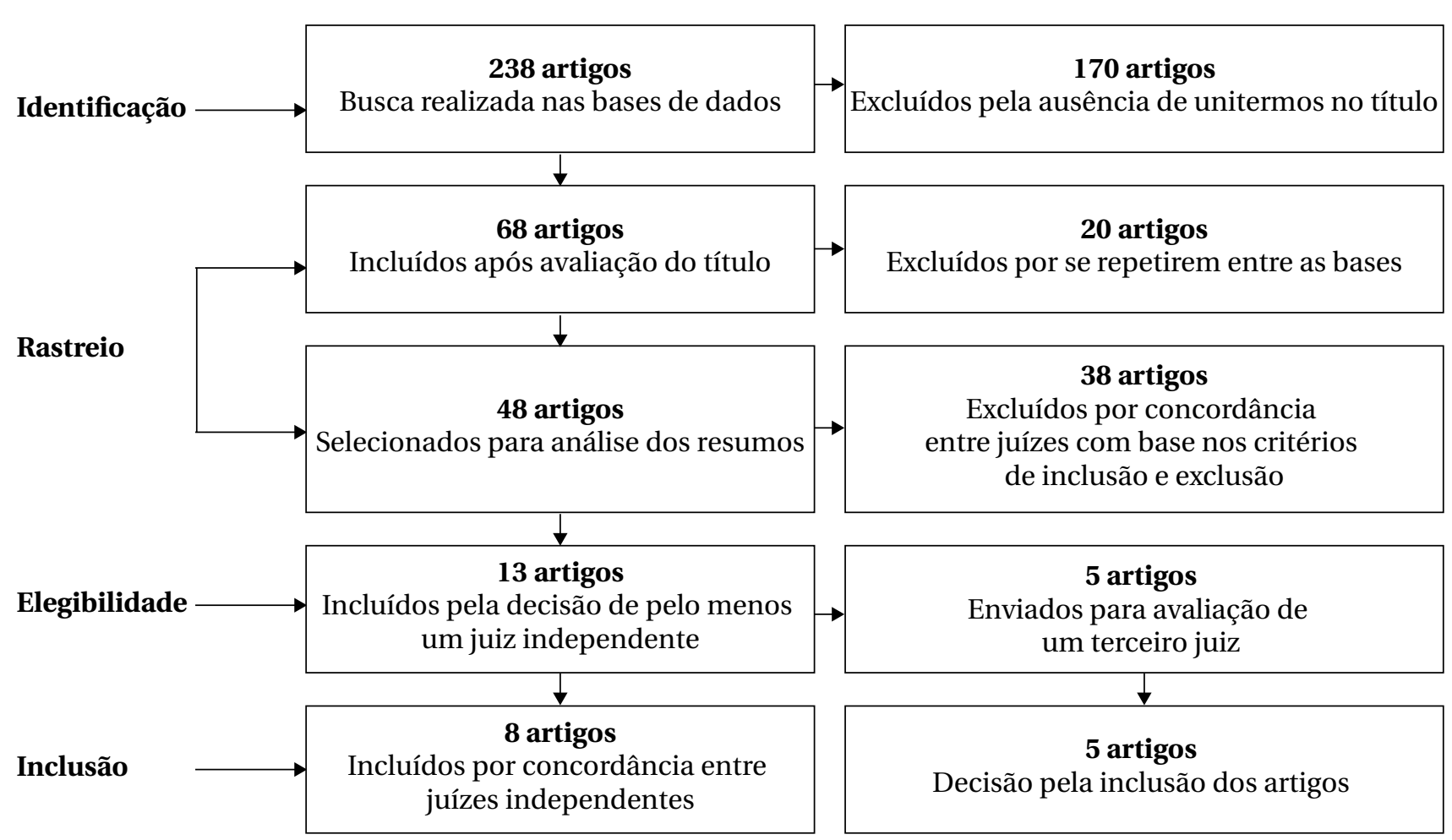

Figura

Fluxograma do levantamento em bases de dados. 
Tabela 1

Caracterização dos Estudos.

\begin{tabular}{|c|c|c|c|c|c|}
\hline Id & Título do Estudo & Ano & País & Delineamento & Aporte teórico \\
\hline 1 & $\begin{array}{l}\text { Resolving attachment injuries in couples } \\
\text { using emotionally focused therapy: steps } \\
\text { toward forgiveness and reconciliation }\end{array}$ & 2006 & Canadá & $\begin{array}{l}\text { Estudo clínico com } \\
\text { grupo controle não } \\
\text { randomizado }\end{array}$ & $\begin{array}{l}\text { Teoria do Apego } \\
\text { Adulto e Abordagem } \\
\text { Sistêmica (Emotional } \\
\text { focused therapy) }\end{array}$ \\
\hline 2 & $\begin{array}{l}\text { Integrating dialectical behavior therapy } \\
\text { and cognitive-behavioral couple therapy: } \\
\text { a couples skills group for emotion } \\
\text { dysregulation }\end{array}$ & 2007 & $\begin{array}{l}\text { Estados } \\
\text { Unidos }\end{array}$ & Estudo de caso & $\begin{array}{l}\text { Cognitivo- } \\
\text { comportamental }\end{array}$ \\
\hline 3 & $\begin{array}{l}\text { Treating emotion dysregulation in a couples } \\
\text { context: a pilot study of a couples skills } \\
\text { group intervention }\end{array}$ & 2007 & $\begin{array}{l}\text { Estados } \\
\text { Unidos }\end{array}$ & $\begin{array}{l}\text { Pré-experimental, } \\
\text { grupo com pré, } \\
\text { pós-teste e follow } \\
\text { up de seis meses }\end{array}$ & $\begin{array}{l}\text { Cognitivo- } \\
\text { comportamental }\end{array}$ \\
\hline 4 & Hats off to problem-solving with couples & 2008 & $\begin{array}{l}\text { Estados } \\
\text { Unidos }\end{array}$ & Estudo de caso & Cognitivo \\
\hline 5 & $\begin{array}{l}\text { Observed communication and associations } \\
\text { with satisfaction during traditional and } \\
\text { integrative behavioral couple therapy }\end{array}$ & 2008 & $\begin{array}{l}\text { Estados } \\
\text { Unidos }\end{array}$ & Observação & Comportamental \\
\hline 6 & $\begin{array}{l}\text { A case study investigating whether the } \\
\text { process of resolving interpersonal problems } \\
\text { in couple therapy is isomorphic to the } \\
\text { process of resolving problems in individual } \\
\text { therapy }\end{array}$ & 2011 & Canadá & Estudo de caso & $\begin{array}{l}\text { Abordagem } \\
\text { Sistêmica (Emotional } \\
\text { focused therapy) }\end{array}$ \\
\hline 7 & $\begin{array}{l}\text { Observed communication in couples } \\
\text { two years after integrative and traditional } \\
\text { behavioral couple therapy: outcome and } \\
\text { link with five-year follow-up }\end{array}$ & 2011 & $\begin{array}{l}\text { Estados } \\
\text { Unidos }\end{array}$ & $\begin{array}{l}\text { Ensaio clínico } \\
\text { randomizado }\end{array}$ & Comportamental \\
\hline 8 & $\begin{array}{l}\text { Partner attachment as a predictor of long- } \\
\text { term response to treatment with couples } \\
\text { therapy }\end{array}$ & 2011 & Holanda & $\begin{array}{l}\text { Estudo clínico } \\
\text { prospectivo, sem } \\
\text { grupo controle }\end{array}$ & $\begin{array}{l}\text { Teorias } \\
\text { comportamental, } \\
\text { sistêmica e } \\
\text { psicodinâmica }\end{array}$ \\
\hline 9 & $\begin{array}{l}\text { The role of specific and common process } \\
\text { variables in residential couple therapy }\end{array}$ & 2011 & Noruega & $\begin{array}{l}\text { Estudo clínico, sem } \\
\text { grupo controle }\end{array}$ & Teoria Sistêmica \\
\hline 10 & $\begin{array}{l}\text { Forgiveness and reconciliation in } \\
\text { emotionally focused therapy for couples: } \\
\text { the client change process and therapist } \\
\text { interventions }\end{array}$ & 2013 & Canadá & $\begin{array}{l}\text { Estudo clínico, sem } \\
\text { grupo controle }\end{array}$ & $\begin{array}{l}\text { Teoria do Apego } \\
\text { Adulto e abordagem } \\
\text { sistêmica (Emotional } \\
\text { focused therapy) }\end{array}$ \\
\hline 11 & $\begin{array}{l}\text { Changes in dyadic communication during } \\
\text { and after integrative and traditional } \\
\text { behavioral couple therapy }\end{array}$ & 2015 & $\begin{array}{l}\text { Estados } \\
\text { Unidos }\end{array}$ & $\begin{array}{l}\text { Ensaio clínico } \\
\text { randomizado }\end{array}$ & Comportamental \\
\hline 12 & $\begin{array}{l}\text { Forgiveness reconciliation and } \\
\text { communication conflict resolution } \\
\text { interventions versus retested controls in } \\
\text { early married couples }\end{array}$ & 2015 & $\begin{array}{l}\text { Estados } \\
\text { Unidos }\end{array}$ & $\begin{array}{l}\text { Ensaio clínico } \\
\text { randomizado }\end{array}$ & $\begin{array}{l}\text { Teorias } \\
\text { comportamental e } \\
\text { sistêmica }\end{array}$ \\
\hline 13 & $\begin{array}{l}\text { The effect of cognitive-behavioral group } \\
\text { marital therapy on marital happiness and } \\
\text { problem solving self-appraisal }\end{array}$ & 2015 & Canadá & $\begin{array}{l}\text { Ensaio clínico } \\
\text { randomizado }\end{array}$ & $\begin{array}{l}\text { Cognitivo- } \\
\text { comportamental }\end{array}$ \\
\hline
\end{tabular}


Tabela 2

Objetivos e principais resultados dos estudos.

\begin{tabular}{ll}
\hline Id. & Objetivos \\
\hline 1 & Descrever como "problemas de \\
& apego" podem se resolver durante \\
& a Terapia Focada na Emoção, \\
& de acordo com o modelo de \\
& resolução de problemas de apego.
\end{tabular}

Principais resultados

15 dos 24 casais resolveram os problemas de apego e nove permaneceram

não resolvidos após o tratamento.

Casais com problemas de apego resolvido apresentaram mais respostas afiliativas (abrir-se e expressar necessidades, validar e compreender)

que respostas hostis/distantes (desmerecimento e culpa, defesa e retirada) e tiveram aumento no ajustamento conjugal e no perdão do pré para o pós-teste. Além disso, ambos os parceiros tiveram níveis mais profundos de experiência se comparados aos casais que não conseguiram resolver os problemas de apego.

Casais com problemas de apego não resolvidos permaneceram dando respostas hostis/distantes. Para estes casais não houve efeito no ajustamento conjugal do pré para o pós-teste.

2 Descrever o desenvolvimento Aumento no ajustamento conjugal.

e a implementação de uma intervenção em grupo baseada na Terapia Cognitivocomportamental Conjugal e na Terapia Comportamental Dialética, apresentando um estudo de caso que ilustra os princípios da intervenção.

3 Desenvolver uma intervenção para casais em que pelo menos um dos parceiros tenha experienciado dificuldades crônicas de regulação emocional.

O casal ganhou confiança de que poderia comunicar-se, resolver problemas e apoiar-se um ao outro enquanto casal.

Melhora nos sintomas depressivos de ambos.

Maior confiança na capacidade de regulação emocional.

4 Demonstrar como usar a técnica de resolução de problemas Bono's Six Thinking Hats (Os Seis Chapéus de Pensamento de Bono) no aconselhamento de casais.

5 Investigar mudanças observadas na comunicação e as mudanças associadas à satisfação em dois tipos de terapias, Terapia de Casal Comportamental Tradicional (Traditional Behavioral Couple Therapy-TBCT) e Terapia de Casal Comportamental Integrativa (Integrative Behavioral Couple Therapy-IBCT).

Parceiros que fizeram o treinamento em Terapia Dialética Comportamental (TDC) reportaram diminuição dos sintomas depressivos do pré para o pósteste. Três de oito participantes também relatam melhora da depressão no follow-up. Houve decréscimo dos escores de raiva do pré-teste para o followup. Não houve diminuição da ansiedade.

Tanto parceiros que participaram do treinamento em TDC quanto os cônjuges que não participaram apresentaram diminuição de afetos positivos do pré para o pós-teste e aumento no follow-up, atingido níveis próximos ao do pré-teste. Treinados em TDC, tiveram redução nos afetos negativos e aumento da regulação emocional do pré-teste para o pósteste, que se manteve no follow-up. Esses efeitos não foram encontrados nos parceiros.

O casal foi capaz de analisar o problema de forma colaborativa, de acordo com diferentes perspectivas, considerando prós, contras e novas possibilidades. Embora em uma sessão não tenha sido possível

chegar a uma decisão, um leque de novas considerações e alternativas foi aberto.

Ambos os tratamentos demonstraram melhoras na comunicação dos casais. Nas interações para resolver problemas do relacionamento, houve diminuição da negatividade, aumento da positividade e da resolução de problemas em homens e mulheres. Em interações sobre problemas pessoais, houve diminuição da negatividade, mas também da positividade e aumento dos comportamentos de retirada.

A TBCT apresentou mais melhora comportamental do que a IBCT, demonstrando maior redução da negatividade para maridos e esposas, e aumento da positividade para os maridos.

Em discussões sobre problemas conjugais, houve relação negativa entre o aumento da negatividade e o aumento da satisfação conjugal, e relação positiva entre o aumento da positividade e da resolução de problemas com o aumento da satisfação conjugal.

O tipo de tratamento recebido não apresentou efeitos na relação entre os comportamentos observados e a satisfação conjugal. 
6 Avaliar se o processo de resolução de problemas interpessoais em terapia de casal é isomorfo ao processo de assimilação em terapia individual e, se possível, estender o modelo para descrever o processo de resolução de problemas interpessoais.

7 Examinar as trajetórias de mudança na comunicação de casais através de um follow up de dois anos e testar se essas mudanças diferem entre duas terapias de casal comportamentais.

$8 \quad$ Examinar o papel do apego ao parceiro como preditor de resultados do tratamento e estudar o efeito potencial do apego durante e após o tratamento.

9 Explorar se há uma relação recíproca ou direta entre variáveis de processos comuns (otimismo, empatia, segurança/confiança, insight) e de processos específicos (comunicação e habilidade de resolução de conflitos) avaliada pelos clientes e pelos terapeutas.

10 Especificar clinicamente o engajamento na sessão de parceiros com problemas de apego (attachment injuries) à medida que completam 0 percurso da resolução dos problemas de apego e alcançam a resolução com sucesso.
Os padrões de mudança no relacionamento interpessoal observados na terapia de casal evoluíram em uma sequência isomórfica às descrições de mudança intrapessoais.

Eventos representando a exclusão de posições relevantes de fala são associadas a relatos de sofrimento psicológico. Já a inclusão de posições antes excluídas é associada a ganhos terapêuticos. Esse diálogo permite que vozes antes excluídas sejam empaticamente compreendidas e incluídas em um processo de tomada de decisões significativas.

A negatividade e os comportamentos de retirada continuaram a diminuir do pós-teste para o follow-up, entretanto, o mesmo aconteceu com a satisfação conjugal, sendo que não houve diferença entre a resolução de conflitos nos dois períodos.

Os níveis e a trajetória de mudança da comunicação após a terapia se relacionaram à satisfação conjugal no follow-up. De maneira geral, a redução da negatividade e comportamentos de retirada e o aumento da positividade e da resolução de conflitos se associaram à maior satisfação conjugal após dois anos.

Casais que apresentaram melhora mais significativa do pré para o pós-teste também apresentaram melhores resultados no follow-up de cinco anos.

Para os pacientes tratados com IBCT, a negatividade das esposas diminuiu, porém, a positividade dos maridos não apresentou diferença significativa do pós-teste para o follow-up de dois anos. Em contrapartida, maridos tratados com TBCT tiveram diminuição da positividade do pós-teste para o follow-up de dois anos e a negatividade das esposas não se alterou no mesmo período.

$\mathrm{O}$ apego inseguro ao parceiro, em termos de modelos disfuncionais de si e dos outros, teve um efeito negativo nos ganhos do tratamento nos 18 meses após a alta da terapia de casal.

As variáveis de processos comuns e específicos, tanto na perspectiva de clientes quanto de terapeutas, melhoraram ao longo do tratamento.

Comunicação e resolução de conflitos foram preditores mais fortes das variáveis de processos comuns do que o inverso.

O insight foi predito pela comunicação e resolução de conflitos reportados por clientes e terapeutas. Já o otimismo foi preditor da comunicação e resolução de conflitos para os clientes, enquanto que, para os terapeutas, a comunicação e a resolução de conflitos foram preditoras do otimismo.

Para os terapeutas, a empatia foi o único preditor com contribuição independente para a comunicação e resolução de conflitos na semana seguinte.

Durante os estágios iniciais (passos 1-4) do AIRM, a forma de cada membro do casal se engajar envolve menos níveis de envolvimento experiencial, modos automáticos de processamento perceptual e respostas não afiliativas. Nos passos subsequentes (5 e 6), novos ciclos de envolvimento relacionados aos problemas de apego são criados, facilitando a execução das tarefas dessa fase. Entretanto, parceiros não resolvidos não se envolvem dessa maneira. Na próxima fase (passos 6 e 7), casais resolvidos continuam envolvidos experiencialmente e emocionalmente na terapia, focando, reavaliando e construindo integrativamente a experiência relacionada ao incidente (que originou o problema). Por fim, o parceiro ofensor responde às necessidades do parceiro ofendido, sendo que o casal é orientado pelo terapeuta a interagir de maneira afiliativa (passo 8). 
Validar empiricamente o Modelo de Resolução de Problemas de Apego (Attachment Injury Resolution Model-AIRM).

Especificar as intervenções de Terapia Focada na Emoção (EFT) associadas à resolução satisfatória de problemas de apego.

11 - Examinar se houve mudança na comunicação durante o período da terapia e do follow up, e se essas mudanças dependiam do tipo de tratamento.

12 Examinar a eficácia de dois tratamentos (Hope-Focused Approach-HOPE, Forgiveness and Reconciliation through Experiencing Empathy-FREE), relativamente aos controles.

13 Avaliar a efetividade geral de um grupo terapêutico cognitivocomportamental para casais em trazer mudanças positivas para a satisfação conjugal e estudar os efeitos desse programa na autoavaliação dos casais sobre suas habilidades de resolução de conflitos.
As intervenções mais frequentemente utilizadas envolviam empatia, validação, acompanhamento e reflexão, ressignificação, reestruturação e modelagem das interações.

A TBCT demonstrou melhora imediata, e superior em relação à IBCT, na comunicação entre os casais. Entretanto, casais tratados com a IBCT apresentaram melhoras após o término do tratamento, o que não ocorreu para os que receberam TBCT.

A comunicação construtiva é mais consistentemente associada a resultados a longo prazo do que a destrutiva.

O maior aumento na positividade e na resolução de problemas do pré para o pós-teste demonstrou associação com a satisfação conjugal.

Apenas o HOPE demonstrou benefícios significativos para o ajustamento diádico, em comparação ao controle.

O FREE melhorou variáveis relacionadas ao perdão, enquanto que o HOPE contribuiu para a melhora de variáveis relacionadas à comunicação.

Ambos os tratamentos melhoraram a empatia.

Em ambas as intervenções, a comunicação negativa aumentou menos do que em relação ao controle. Além disso, o aumento na comunicação positiva foi maior para os participantes do tratamento do que os casais do grupo controle.

Houve uma redução moderada dos níveis de cortisol após o tratamento, mas em ambas as intervenções, o efeito diminuiu após 12 meses.

As melhoras reportadas pelos casais no grupo de tratamento foram superiores às do grupo controle, confirmando que a participação no grupo aumentou a autoeficácia percebida em relação à resolução de conflitos.

Após a intervenção, os participantes reportaram uma melhora significativa na capacidade de confrontar diferentes atividades de resolução de problemas e da confiança em suas capacidades de resolução de conflitos. Os casais reportaram maior ajustamento conjugal após o programa, independente da avaliação inicial de suas habilidades de resolução de conflitos. ram resultados inconclusivos ou referiram que, apesar da superioridade da TBCT ao longo do tratamento, os pacientes tratados com a IBCT apresentaram melhoras após o término do tratamento, o que não ocorreu com os pacientes tratados com a TBCT.

Os estudos baseados nas teorias sistêmica e psicodinâmica abrangeram aspectos relativos ao processo psicoterapêutico e a variáveis de resultado. Foram identificados padrões de mudança interpessoais semelhantes aos intrapessoais e intervenções envolvendo validação, ressignificação, reestruturação e modelagem de interações. Quanto às variáveis de resultado, foram reportadas melhoras no ajustamento conjugal, na capacidade de expressar necessidades e empatia, na comunicação e na resolução dos confli- tos. Além disso, houve diminuição das respostas hostis entre o casal durante a terapia.

\section{Discussão}

O ano de publicação dos estudos encontrados não estão distribuídos igualmente entre 2006-2015, período delimitado na busca. Não houve publicações nos anos de 2009, 2010, 2012 e 2014, porém, oito dos 13 estudos foram publicados entre 2011 e 2015. Esse resultado pode indicar uma necessidade e preocupação crescente dos pesquisadores em desenvolver estudos sobre as psicoterapias baseadas em evidências a respeito das diversas temáticas que envolvem a conjugalidade e, entre elas, as estratégias de resolução de conflito ainda necessi- 
tam ser aprofundadas (Brum et al., 2012; Enéas, 2008; Peuker et al., 2009; Serralta et al., 2011).

Os Estados Unidos foram o lugar com o maior número de publicações, seguidos do Canadá, Noruega e Holanda. A concentração de estudos sobre a temática na América do Norte indica maior investimento desses países em pesquisas científicas, que, inevitavelmente, são utilizadas como critério de comparação em outros contextos. Conforme apontado no estudo de Costa et al. (2016), a predominância de estudos científicos no contexto norte-americano demonstra a influência que outros países recebem da referida "cultura científica" e aponta a necessidade de investimento em pesquisa por parte de outros países. No Brasil, por exemplo, não foi encontrada nenhuma publicação sobre pesquisa em psicoterapia de casal e estratégias de resolução de conflito nos últimos dez anos.

A predominância de ensaios clínicos randomizados entre os estudos investigados pode indicar a preocupação dos pesquisadores em aumentar o rigor metodológico por meio de estudos de eficácia. Embora os ensaios clínicos sem grupo controle, denominados estudos de efetividade, os estudos de caso e os estudos de observação tenham sido menos frequentes, são investigações que também possibilitam avanços em termos de conhecimento científico.

Os estudos experimentais garantem maior controle de variáveis e possibilitam a criação de protocolos e manuais para o tratamento de determinadas psicopatologias e o contexto terapêutico é criado em "laboratório". Já os estudos quase-experimentais ou de efetividade, os estudos de caso e os estudos de observação alcançam maior validade externa por produzirem situações reais e obterem resultados semelhantes aos da prática profissional na clínica psicológica. As limitações desses delineamentos são a interferência de variáveis ambientais, do terapeuta e do paciente que nem sempre são possíveis controlar (Brum et al., 2012; Peuker et al., 2009; Serralta et al., 2011).

As perspectivas teóricas dos estudos foram predominantemente comportamentais e cognitivo-comportamentais (Baucom, Baucom, \& Christensen, 2015; Baucom, Sevier, Eldridge, Doss, \& Christensen, 2011; Bélanger, Laporte, Sabourin, \& Wright, 2015; Kirby \& Baucom, 2007a; 2007b; Li, Lin, Nelson, \& Eckstein, 2008; Sevier, Eldridge, Jones, Doss, \& Christensen, 2008) em detrimento das abordagens sistêmicas, teoria do apego e teoria psicodinâmica (Conradi, Jonge, Neeleman, Simons, \& Sytema, 2011; Makinen, \& John- son, 2006; Schielke et al., 2011; Tilden, Hoffart, Sexton, Finset, \& Gude, 2011; Zuccarini, Johnson, Dalgleish, \& Makinen, 2013). Esse resultado pode estar associado ao fato de as primeiras abordagens estarem mais próximas dos pressupostos positivistas, quantitativos em ciência, que podem ser comparados à utilização de protocolos e manuais. Além disso, observa-se o rigor metodológico dos estudos nas abordagens comportamentais e cognitivo-comportamentais que utilizaram, predominantemente, ensaios clínicos randomizados (Baucom et al., 2011; 2015; Bélanger et al., 2015).

As pesquisas em psicoterapia de casal também foram o foco de investigação em outras abordagens teóricas, como a teoria do apego e a teoria psicodinâmica (Conradi et al., 2011; Makinen, \& Johnson, 2006; Zuccarini et al., 2013). Evidencia-se que pesquisadores dessas áreas têm buscado evidências de validade sobre a eficácia e/ou efetividade das teorias e das técnicas psicoterápicas, o que pode estar associado à preocupação crescente com a consolidação da Psicologia enquanto ciência em diferentes abordagens teóricas (Serralta et al., 2011).

A combinação entre abordagens teóricas também parece ser uma tendência (Conradi et al., 2011; Makinen, \& Johnson, 2006; Worthington Jr et al., 2015; Zuccarini et al., 2013), especialmente entre a abordagem sistêmica, por meio da Terapia Focada na Emoção, e a Teoria do Apego (Makinen, \& Johnson, 2006; Zuccarini et al., 2013). Ainda que as duas perspectivas sejam distintas, essa proposta de aproximação aponta a necessidade de avaliar se existe associação entre a Terapia Focada na Emoção e a Teoria do Apego e de que forma isso se expressa na terapia e no relacionamento dos casais em tratamento.

Nos estudos em que abordagens teóricas foram comparadas e analisadas (Kirby, \& Baucom, 2007a; Worthington Jr et al., 2015), foi demonstrado que os casais obtiveram ganhos após a realização do tratamento, tanto através de medidas pré e pós-tratamento e de follow-up, como de comparação entre grupo experimental e grupo controle. Esse resultado apenas corrobora o veredito Dodô, de que as diferentes psicoterapias funcionam, independentemente de como (Brum et al., 2012).

Foram identificadas distintas variáveis associadas à resolução dos conflitos conjugais durante o processo terapêutico, descritas como: problemas de apego (Makinen, \& Johnson, 2006), dificuldade crônica de regulação emocional (Kirby, \& Baucom, 2007b), técnica 
específica de resolução de problemas (Li et al., 2008), manejo de estratégias em terapia de casal e em terapia individual (Schielke et al., 2011), função do apego ao parceiro nos resultados do tratamento (Conradi et al., 2011), relação entre processos comuns e processos específicos (Tilden et al., 2011), satisfação conjugal e autoavaliação de habilidades de resolução de problemas (Bélanger et al., 2015). Essa diversidade de abordagens e variáveis ilustra a complexidade do fenômeno e a necessidade de aprimorar cada vez mais as pesquisas sobre a conjugalidade, a fim de construir um corpus de conhecimento mais consistente sobre a temática (Costa et al., 2016).

Além disso, quatro estudos focaram abordagem teórica e temáticas associadas à resolução dos conflitos (Baucom et al., 2011; 2015; Sevier et al., 2008; Zuccarini et al., 2013). Três estudos envolveram a abordagem comportamental e a variável comunicação (Baucom et al., 2011; 2015; Sevier et al., 2008), enquanto um estudo envolveu mais de uma abordagem e os problemas de apego (Zuccarini et al., 2013). Outras variáveis investigadas foram a satisfação conjugal (Sevier et al., 2008) e a resolução de conflitos (Baucom et al., 2011). A comparação entre diferentes terapias e a análise da comunicação entre os cônjuges já era investigada em estudos anteriores à última década (Baucom, 1982; Behrens et al., 1990; Braukhaus et al., 2003; Sayers et al., 1991). Em contrapartida, nota-se que outras abordagens (Zuccarini et al., 2013) têm investido na consolidação de modelos e técnicas por meio de evidências de validade empíricas (Brum et al., 2012; Enéas, 2008; Peuker et al., 2009).

Com relação aos resultados dos estudos, três utilizaram abordagem sistêmica, por meio da Terapia Focada na Emoção, e apresentaram resultados associados ao apego (Conradi et al., 2011; Makinen, \& Johnson, 2006; Zuccarini et al., 2013). O apego inseguro provocou efeitos negativos à relação conjugal e durante a terapia (Conradi et al., 2011), porém, os problemas relacionados ao apego foram passíveis de resolução (Makinen, \& Johnson, 2006; Zuccarini et al., 2013). A resolução desses problemas parece ser facilitada pela emissão de respostas afiliativas, expressão de necessidades, validação e compreensão do parceiro. Os cônjuges iniciam o processo de terapia com respostas reativas e não afiliativas, entram em contato com os problemas de apego nos estágios intermediários e se conectam experiencialmente com as suas emoções nos estágios finais, reavaliando e ressignificando a experiência (Zuccarini et al., 2013). A resolução desses problemas tende a aumentar o ajustamento conjugal e a capacidade de perdoar dos parceiros. Contudo, respostas hostis ou distantes, por meio de desmerecimento, culpabilização, defesa ou retirada, podem dificultar esse processo (Makinen, \& Johnson, 2006).

Assim, padrões comportamentais e interacionais aprendidos com as figuras parentais parecem estar associados ao ajustamento conjugal e à capacidade de resolução de problemas pelo casal (Curran et al., 2011). Considerando que os estilos de apego disfuncionais com a família de origem foram transferidos para a relação conjugal e que a díade é uma unidade que se retroalimenta e pode ter dificuldades emocionais semelhantes, a psicoterapia de casal seria fortemente indicada, pois os processos de mudança poderiam ocorrer em nível individual e diádico (Costa et al., 2016; Scheeren et al., 2015).

A comunicação entre o casal foi a variável de resultado em cinco estudos investigados (Baucom et al., 2011; 2015; Sevier et al., 2008; Tilden et al., 2011; Worthington Jr et al., 2015). Além da melhora na comunicação, as intervenções aplicadas nesses estudos também aumentaram os níveis de satisfação (Baucom et al., 2011; 2015; Sevier et al., 2008) e aprimoraram as habilidades de resolução de conflitos dos casais (Sevier et al., 2008; Tilden et al., 2011). A comunicação apresenta-se como uma importante variável de análise na conjugalidade, provocando alterações em nível individual, como na satisfação com o casamento, e diádico, na resolução de conflitos. A comunicação deve ainda ser observada pelos profissionais da área clínica, considerando que padrões comunicacionais negativos tendem a se sobressair aos positivos e podem cristalizar a dinâmica conjugal, dificultando a mudança de comportamentos e da interação conjugal ao longo do tempo (Markman et al., 2010).

A positividade e a negatividade dos cônjuges durante os conflitos também parecem se associar aos resultados das interações do casal. A primeira está relacionada à resolução construtiva dos conflitos, e a segunda, à diminuição nos níveis de satisfação conjugal (Baucom et al., 2011; Sevier et al., 2008). A empatia (Tilden et al., 2011; Worthington Jr et al., 2015), o otimismo (Tilden et al., 2011), o ajustamento diádico e o perdão (Worthington Jr et al., 2015), por sua vez, também tiveram um impacto importante nos resultados da terapia. Considerando que a positividade e a 
negatividade emergem da interação, a comunicação, verbal e não verbal, novamente aparece como um fator importante na psicoterapia. No mesmo sentido, a capacidade de ser empático, de perdoar e de ser otimista, associa-se ao clima conjugal, que poderá ser de maior positividade ou negatividade dependendo de fatores individuais e diádicos (Costa et al., 2016). Essa compreensão vai ao encontro da ideia de que, na terapia de casal, as mudanças relacionais e intrapessoais estão associadas (Schielke et al., 2011).

Por fim, o ajustamento conjugal ou ajustamento diádico é citado como variável de resultado em três estudos (Bélanger et al., 2015; Kirby, \& Baucom, 2007b; Worthington Jr et al., 2015). As intervenções também melhoraram a comunicação, a empatia, o perdão (Worthington Jr et al., 2015), a confiança (Bélanger at al., 2015), a regulação emocional, a confiança, o apoio (Kirby, \& Baucom, 2007b) e a resolução de problemas (Bélanger et al., 2015; Kirby \& Baucom, 2007b). A regulação emocional do afeto positivo e negativo, da ansiedade e da raiva foi apontada como variável de resultado em outro estudo de Kirby e Baucom (2007a) para a diminuição dos sintomas depressivos.

No estudo de Li et al. (2008), a utilização da técnica para resolução de conflitos Bono's Six Thinking Hats possibilitou aos cônjuges analisar os conflitos de forma colaborativa. Entretanto, as informações fornecidas pelos autores quanto ao procedimento utilizado e ao contexto de aconselhamento conjugal dificultou a compreensão da técnica e dos resultados. Isso pode ter ocorrido devido à especificidade da proposta, ainda pouco conhecida e difundida na área.

Finalmente, identifica-se, nos resultados dos estudos desta revisão, uma importante contribuição da terapia de casal para o aprimoramento da comunicação, da empatia, da capacidade de perdoar, apoiar e confiar no cônjuge, bem como para o desenvolvimento de estratégias de resolução dos conflitos conjugais. Tais aspectos estão fortemente documentados na literatura científica (Anderson, \& Johnson, 2010; Leggett et al., 2012; Markman et al., 2010; Schoebi et al., 2012; Sierau, \& Herzberg, 2012; Sullivan et al., 2010; Veldorale-Brogan et al., 2013; Wheeler et al., 2010; Whiting, 2008). Além disso, fatores como regulação emocional são referidos por grupos de pesquisadores com significativa trajetória em pesquisa (Baucom, 1982; Baucom et al., 2011; 2015; Kirby \& Baucom, 2007a; 2007b; Sayers et al., 1991), indicando que se trata de uma área de conhecimento que necessita ser explorada considerando as diferentes perspectivas teóricas e variáveis implicadas nesse processo.

\section{Conclusão}

Este estudo teve como objetivo revisar a literatura científica sobre psicoterapia de casal e estratégias de resolução de conflito, e identificar variáveis envolvidas no processo terapêutico. As principais abordagens teóricas encontradas foram a comportamental, a cognitivo-comportamental, a sistêmica e a psicodinâmica. As duas primeiras empregaram técnicas mais diretivas em relação às outras duas, além de utilizar predominantemente ensaios clínicos randomizados, enquanto nas abordagens sistêmica e psicodinâmica, os estudos clínicos sem grupo controle foram mais frequentes. As demandas apresentadas pelos casais que participaram das intervenções não foram relatadas. Além disso, o fato de os estudos avaliarem os resultados da psicoterapia ou compararem diferentes técnicas e abordagens, em detrimento dos processos psicoterápicos, dificultou a análise da influência das diferentes perspectivas teóricas, técnicas e demandas nesses processos interventivos.

Nesse sentido, ressalta-se a importância de os autores proporcionarem uma descrição mais detalhada dos participantes, das formas de recrutamento e das demandas apresentadas pelos casais ao buscar psicoterapia. Essa medida possibilitaria analisar se determinadas técnicas, ou mesmo abordagens teóricas, são mais indicadas para tipos específicos de demanda. Além disso, os participantes que buscam a psicoterapia espontaneamente poderiam ser comparados àqueles que respondem aos anúncios de recrutamento para as pesquisas, a fim de se verificar as possíveis diferenças entre a aplicação das técnicas no contexto de pesquisa em relação à prática cotidiana na clínica psicológica.

As pesquisas em psicoterapia de casal apresentaram também pontos comuns entre si e com os estudos que enfocam as relações e os conflitos conjugais. Por um lado, há consenso sobre o papel da comunicação, da empatia, do perdão, do apoio mútuo, da confiança e da resolução de conflitos na conjugalidade. Essas variáveis são fundamentais para a compreensão do fenômeno, sendo que os terapeutas devem estar atentos à forma como são expressas pelos casais no setting terapêutico. Por outro lado, há falta de homogeneidade e de clareza nos termos utilizados, por exemplo, "positividade" e "negatividade". Essas inconsistências tornam a temática, que é intrinsecamente complexa, 
ainda mais difícil de ser estudada de forma sistemática e coerente, dificultando o desenvolvimento de conhecimentos consistentes sobre o tema.

Ressalta-se o fato de que não foram encontrados estudos clínicos sobre terapia de casal realizados no Brasil nos últimos 10 anos, indicando que o estudo da temática ainda é incipiente no País. É possível que isso ocorra uma vez que a realização desse tipo de pesquisa necessita recursos financeiros, humanos e espaço físico, os quais nem sempre os pesquisadores brasileiros dispõem. Porém, destaca-se a importância da caracterização dos casais que buscam atendimento, das abordagens e técnicas mais utilizadas pelos psicoterapeutas, e dos resultados alcançados com as intervenções. A adesão ao tratamento, os processos psicoterapêuticos e os resultados obtidos podem variar em função da formação dos terapeutas e das características culturais, que são diferentes no Brasil em comparação aos países norte-americanos.

Assim, os resultados apresentados neste estudo podem ser úteis para os pesquisadores que buscam realizar investigações sobre o tema, fornecendo um panorama a respeito do estado da arte na literatura internacional, apontando consensos já estabelecidos e inconsistências que devem ser superadas. Psicoterapeutas também podem se beneficiar desta revisão, que apresenta as abordagens e técnicas utilizadas em outros contextos, discute o papel de diferentes técnicas e variáveis na psicoterapia e proporciona uma fonte de consulta sobre a efetividade dessas técnicas enquanto pesquisas nacionais não são realizadas.

Por fim, algumas limitações deste estudo podem ser apontadas. $\mathrm{O}$ recorte em relação às palavras-chave utilizadas e as bases de dados em que as buscas foram realizadas pode ter limitado o alcance da totalidade dos trabalhos sobre o tema investigado. Além disso, foram utilizados critérios de exclusão específicos, que descartaram estudos que tivessem como foco psicopatologias ou que tratassem de programas de psicoeducação e prevenção. Apesar desse critério contribuir para a homogeneidade entre os estudos analisados, é possível que técnicas úteis no treinamento de habilidades de resolução de conflitos não tenham sido incluídas na revisão. Além disso, a inclusão de outros tipos de estudo, como artigos teóricos, poderia contribuir para analisar os processos psicoterapêuticos, pouco abordados nos artigos empíricos, junto aos resultados relacionados à efetividade dos tratamentos. Assim, sugere-se que estudos futuros analisem esses aspectos de forma integrada, a fim de proporcionar uma visão ainda mais completa sobre o fenômeno.

\section{Referências}

Anderson, S. R., \& Johnson, L. N. (2010). A dyadic analysis of the between and within system alliances on distress. Family Process, 49(2), 220-235. https://doi.org/10.1111/j.1545-5300.2010.01319.x

Baucom, D. H. (1982). A comparison of behavioral contracting and problem-solving/communications training in behavioral marital therapy. Behavior Therapy, 13(2), 162-174. https://doi.org/10.1016/S0005-7894(82)80060-9

Baucom, K. J., Baucom, B. R., \& Christensen, A. (2015). Changes in dyadic communication during and after integrative and traditional behavioral couple therapy. Behavior Research and Therapy, 65, 18-28. https://doi.org/10.1016/j.brat.2014.12.004

Baucom, K. J., Sevier, M., Eldridge, K. A., Doss, B. D., \& Christensen, A. (2011). Observed communication in couples two years after integrative and traditional behavioral couple therapy: outcome and link with five-year follow-up. Journal of Consulting and Clinical Psychology, 79(5), 565-576. https://doi.org/10.1037/a0025121

Behrens, B. C., Sanders, M. R., \& Halford, W. K. (1990). Behavioral marital therapy: an evaluation of treatment effects acrosshigh andlowrisksettings. BehaviorTherapy, 21(4),423-433.https://doi.org/10.1016/S0005-7894(05)80356-9

Bélanger, C., Laporte, L., Sabourin, S., \& Wright, J. (2015). The effect of cognitive-behavioral group marital therapy on marital happiness and problem solving self-appraisal. The American Journal of Family Therapy, 43(2), 103-118. https://doi.org/10.1080/01926187.2014.956614

Braukhaus, C., Hahlweg, K., Kroeger, C., Groth, T., \& Fehm-Wolfsdorf, G. (2003). The effects of adding booster sessions to a prevention-training program for committed couples. Behavioral and Cognitive Psychotherapy, 31(03), 325-336. https://doi.org/10.1017/S1352465803003072

Brum,E.H.M.,Frizzo, G. B., Gomes,A.G.,Silva,M.R.,Souza,D.D.,\&Piccinini,C.A.(2012).Evoluçãodosmodelosdepesquisa em psicoterapia. Estudos dePsicologia (Campinas),29(2), 259-269. https://doi.org/10.1590/S0103-166X2012000200012 
Carlson, J., \& Dinkmeyer, D. (1987). Adlerian marriage therapy. The American Journal of Family Therapy, 15(4), 326-332. https://doi.org/10.1080/01926188708250692

Conradi, H. J., Jonge, P., Neeleman, A., Simons, P., \& Sytema, S. (2011). Partner attachment as a predictor of long-term response to treatment with couples therapy. Journal of Sex \& Marital Therapy, 37(4), 286-297. https://doi.org/10.1080/0092623X.2011.582435

Costa, C. B., Cenci, C. M. B., \& Mosmann, C. P. (2016). Conflito conjugal e estratégias de resolução: Uma revisão sistemática da literatura. Temas em Psicologia, 24(1), 325-38. https://doi.org/10.9788/TP2016.1-22

Costa, A. B., Zoltowski, A. P. C., Koller, S. H., \& Teixeira, M. A. P. (2015). Construção de uma escala para avaliar a qualidade metodológica de revisões sistemáticas. Ciências \& Saúde Coletiva, 20(8), 2441-2452. https://doi.org/10.1590/1413-81232015208.10762014

Curran, M., Ogolsky, B., Hazen, N., \& Bosch, L. (2011). Understanding marital conflict 7 years later from prenatal representations of marriage. Family Process, 50(2), 221-234. https://doi.org/10.1111/j.1545-5300.2011.01356.x

Davidson, G. N., \& Horvath, A. O. (1997). Three sessions of brief couples therapy: a clinical trial. Journal of Family Psychology, 11(4), 422-435. https://doi.org/10.1037/0893-3200.11.4.422-435

Enéas, M. L. E. (2008). Pesquisas em psicoterapia: seções especiais de periódico (1981 a 1994). Psicologia: Teoria e Pesquisa, 24(1), 111-116. https://doi.org/10.1590/S0102-37722008000100013

Fincham, F. D. (2003). Marital conflict correlates, structure, and context. Current Directions in Psychological Science, 12(1), 23-27. https://doi.org/10.1111/1467-8721.01215

Goldman, A., \& Greenberg, L. (1992). Comparison of integrated systemic and emotionally focused approaches to couples therapy. Journal of Consulting and Clinical Psychology, 60(6), 962-969. https://doi.org/10.1037/0022-006X.60.6.962

Jacobson, N. S. (1984). A component analysis of behavioral marital therapy: the relative effectiveness of behavior exchange and communication/problem-solving training. Journal of Consulting and Clinical Psychology, 52(2), 295-305. https://doi.org/10.1037/0022-006X.52.2.295

Jacobson, N. S. (1979). Increasing positive behavior in severely distressed marital relationships: the effects of problem-solving training. Behavior Therapy, 10(3), 311-326. https://doi.org/10.1016/S0005-7894(79)80021-0

Jacobson, N.S. (1978). Specific and nonspecific factors in the effectiveness of a behavioral approach to the treatment of marital discord. Journal of Consulting and Clinical Psychology, 46(3), 442-452. https://doi.org/10.1037/0022-006X.46.3.442

Jacobson, N. S., Follette, V. M., Follette, W. C., Holtzworth-Munroe, A., Katt, J. L., \& Schmaling, K. B. (1985). A component analysis of behavioral marital therapy: 1-year follow-up. Behavior Research and Therapy, 23(5), 549-555. https://doi.org/10.1016/0005-7967(85)90102-0

Jacobson, N. S., Schmaling, K. B., \& Holtzworth-Munroe, A. (1987). Component analysis of behavioral marital therapy: 2-year follow-up and prediction of relapse. Journal of Marital and Family Therapy, 13(2), 187-195. https:// doi.org/10.1111/j.1752-0606.1987.tb00696.x

Johnson, S. M., \& Greenberg, L. S. (1985). Differential effects of experiential and problem-solving interventions in resolving marital conflict.JournalofConsultingand ClinicalPsychology, 53(2),175-184.https://doi.org/10.1037/0022-006X.53.2.175

Kaiser, A., Hahlweg, K., Fehm-Wolfsdorf, G., \& Groth, T. (1998). The efficacy of a compact psychoeducational group-training program for married couples. Journal of Consulting and Clinical Psychology, 66(5), 753-760. https:// doi.org/10.1037/0022-006X.66.5.753

Kirby, J. S., \& Baucom, D. H. (2007a). Integrating dialectical behavior therapy and cognitive-behavioral couple therapy: a couples skills group for emotion dysregulation. Cognitive and Behavioral Practice, 14(4), 394-405. https://doi.org/10.1016/j.cbpra.2006.09.006

Kirby,J.S.,\&Baucom,D.H.(2007b).Treatingemotiondysregulationinacouplescontext:apilotstudyofacouplesskillsgroup intervention. Journal of Marital and Family Therapy, 33(3), 375-391. https://doi.org/10.1111/j.1752-0606.2007.00037.x

Leggett, D. G., Roberts-Pittman, B., Byczek, S., \& Morse, D.T. (2012). Cooperation, conflict, and marital satisfaction: Bridging theory, research, and practice. The Journal of Individual Psychology, 68(2), 182-199. Retrieved from http://eds.b.ebscohost. com/eds/pdfviewer/pdfviewer?sid=22de6a9f-e52c-4cbd-b5dc-9d282ffe0e70\%40sessionmgr114\&vid=5\&hid=121

Li, C. S., Lin, Y. F., Nelson, J., \& Eckstein, D. (2008). Hats off to problem solving with couples. The Family Journal, 16(3), 254-257. https://doi.org/10.1177/1066480708317674 
Makinen, J. A., \& Johnson, S. M. (2006). Resolving attachment injuries in couples using emotionally focused therapy: steps toward forgiveness and reconciliation. Journal of Consulting and Clinical Psychology, 74(6), 10551064. https://doi.org/10.1037/0022-006X.74.6.1055

Markman, H. J., Rhoades, G. K., Stanley, S. M., Ragan, E. P., \&Whitton, S. W. (2010). The premarital communication roots of marital distress and divorce: the first five years of marriage. Journal of Family Psychology, 24(3), 289-298. https://doi.org/10.1037/a0019481

Mitchell, H. E., Bullard, J.W., \& Mudd, E. H. (1962). Areas of marital conflict in successfully and unsuccessfully functioning families. Journal of Health and Human Behavior, 3(2), 88-93. https://doi.org/10.2307/2948928

Mosmann, C. P., \& Falcke, D. (2011). Conflitos conjugais: motivos e frequência. Revista da SPAGESP, 12(2), 5-16. Recuperado de http://pepsic.bvsalud.org/pdf/rspagesp/v12n2/v12n2a02.pdf

Paleari, F. G., Regalia, C., \& Fincham, F. D. (2010). Forgiveness and conflict resolution in close relationships: within and cross partner effects. Universitas Psychologica, 9(1), 35-56. Retrieved from http://www.scielo.org.co/pdf/rups/v9n1/v9nla04.pdf

Peuker, A. C., Habigzang, L. F., Koller, S. H., \& Araujo, L. B. (2009). Avaliação de processo e resultado em psicoterapias: uma revisão. Psicologia em Estudo, 14(3), 439-445. https://doi.org/10.1590/S1413-73722009000300004

Rohrbaugh, M. J., \& Shoham, V. (2001). Brief therapy based on interrupting ironic processes: the Palo Alto model. Clinical Psychology: Science and Practice, 8(1), 66-81. https://doi.org/10.1093/clipsy.8.1.66

Sampaio, R. F., \& Mancini, M. C. (2007). Estudos de revisão sistemática: um guia para síntese criteriosa da evidência científica. Revista Brasileira de Fisioterapia, 11(1), 83-89. https://doi.org/10.1590/S1413-35552007000100013

Sayers, S. L., Baucom, D. H., Sher, T. G., \&Weiss, R. L. (1991). Constructive engagement, behavioral marital therapy, and changes in marital satisfaction. Behavioral Assessment, 13(1), 25-49. Retrieved from https://www.academia. edu/20451225/Constructive_engagement_behavioral_marital_therapy_and_changes_in_marital_satisfaction

Scheeren, P., Delatorre, M. Z., Neumann, A. P., \&Wagner, A. (2015). O papel preditor dos estilos de apego na resolução do conflito conjugal. Estudos e Pesquisas em Psicologia, 15(3), 835-852. https://doi.org/10.12957/epp.2015.19415

Schielke, H. J., Stiles, W. B., Cuellar, R. E., Fishman, J. L., Hoener, C., Del Castillo, D., et al. (2011). A case study investigating whether the process of resolving interpersonal problems in couple therapy is isomorphic to the process of resolving problems in individual therapy. Pragmatic Case Studies in Psychotherapy, 7(4), 477-528. https://doi.org/10.14713/pcsp.v7i4.1114

Schoebi, D., Karney, B. R., \& Bradbury, T. N. (2012). Stability and change in the first 10 years of marriage: Does commitment confer benefits beyond the effects of satisfaction? Journal of Personality and Social Psychology, 102(4), 729-742. https://doi.org/10.1037/a0026290

Serralta, F.B., Nunes, M.L.T., \&Eizirik, C.L. (2011).Consideraçõesmetodológicas sobreo estudodecasonapesquisa em psicoterapia.EstudosdePsicologia(Campinas),28(4),501-510.https://doi.org/10.1590/S0103-166X2011000400010

Sevier, M., Eldridge, K., Jones, J., Doss, B. D., \& Christensen, A. (2008). Observed communication and associations with satisfaction during traditional and integrative behavioral couple therapy. Behavior Therapy, 39(2), 137-150. https://doi.org/10.1016/j.beth.2007.06.001

Sierau, S., \& Herzberg, P.Y. (2012). Conflict resolution as a dyadic mediator: Considering the partner perspective on conflict resolution. European Journal of Personality, 26(3), 221-232. https://doi.org/10.1002/ per.828

Sullivan, K. T., Pasch, L. A., Johnson, M. D., \& Bradbury, T. N. (2010). Social support, problem solving, and the longitudinal course of newlywed marriage. Journal of Personality and Social Psychology, 98(4), 631-644. https://doi.org/10.1037/a0017578

Tilden, T., Hoffart, A., Sexton, H., Finset, A., \& Gude, T. (2011). The role of specific and common process variables in residential couple therapy. Journal of Couple \& Relationship Therapy, 10(3), 262-278. https://doi.org/10.1080/15332691.2011.588100

Veldorale-Brogan, A., Lambert, N. M., Fincham, F. D., \& DeWall, C. N. (2013). The virtue of problem solving: perceived partner virtues as predictors of problem-solving efficacy. Personal Relationships, 20(3), 511-523. https://doi.org/10.1111/j.1475-6811.2012.01421.x

Wheeler,L.A.,Updegraff,K.A.,\&Thayer,S.M.(2010).Conflictresolutioninmexican-origincouples:culture, gender, and marital quality. Journal of Marriage and Family, 72(4), 991-1005. https://doi.org/10.1111/j.1741-3737.2010.00744.x

Whiting, J. B. (2008). The role of appraisal distortion, contempt, and morality in couple conflict: a grounded theory. Journal of Marital and Family Therapy, 34(1), 44-57. https://doi.org/10.1111/j.1752-0606.2008.00052.x 
Worthington Jr, E. L., Berry, J. W., Hook, J. N., Davis, D. E., Scherer, M., Griffin, B. J., et al. (2015). Forgiveness reconciliation and communication conflict resolution interventions versus retested controls in early-married couples. Journal of Counseling Psychology, 62(1), 14-27. https://doi.org/10.1037/ cou0000045

Zuccarini, D., Johnson, S. M., Dalgleish, T. L., \& Makinen, J. A. (2013). Forgiveness and reconciliation in emotionally focused therapy for couples: The client change process and therapist interventions. Journal of Marital and Family Therapy, 39(2), 148-162. https://doi.org/10.1111/j.1752-0606.2012.00287.x

\section{Crístofer Batista da Costa}

Doutorando pela Universidade do Vale do Rio dos Sinos, São Leopoldo - RS, Brasil.

E-mail: cristoferbatistadacosta@gmail.com

Marina Zanella Delatorre

Doutoranda pela Universidade Federal do Rio Grande do Sul, Porto Alegre - RS, Brasil.

E-mail: marina_mzd@yahoo.com.br

\section{Adriana Wagner}

Doutora em Psicologia pela Universidade Federal do Rio Grande do Sul, Porto Alegre - RS, Brasil.

E-mail: adrianawagner.ufrgs@hotmail.com

\section{Clarisse Pereira Mosmann}

Doutora em Psicologia pela Universidade do Vale do Rio dos Sinos, São Leopoldo - RS, Brasil.

E-mail: clarissemosmann@gmail.com

Endereço para envio de correspondência:

Universidade do Vale do Rio dos Sinos - Unisinos. Av. Unisinos, 950. Bairro Cristo Rei. CEP: 93.022-000.

São Leopoldo - RS. Brasil.

Recebido 14/02/2016

Aprovado 16/11/2016

Received 02/14/2016

Approved 11/16/2016

Recibido 14/02/2016

Aceptado 16/11/2016

Como citar: Costa, C. B., Delatorre, M. Z., Wagner, A., \& Mosmann, C. P. (2017). Terapia de casal e estratégias de resolução de conflito: uma revisão sistemática. Psicologia: Ciência e Profissão, 37(1): 208-223. https://doi.org/10.1590/1982-3703000622016

How to cite: Costa, C. B., Delatorre, M.Z., Wagner, A., \& Mosmann, C. P. (2017). Couple therapy and conflict resolution strategies: a systematic review. Psicologia:Ciênciae Profissão, 37(1):208-223. https://doi.org/10.1590/1982-3703000622016

Cómo citar: Costa, C. B., Delatorre, M. Z., Wagner, A., \& Mosmann, C. P. (2017). Terapia de pareja y estrategias de resolución de conflicto: una revisión sistemática. Psicologia: Ciência e Profissão, 37(1): 208-223. https://doi.org/10.1590/1982-3703000622016 